\title{
Broadband Dual Circularly Polarized Magnetoelectric Dipole Antenna Fed by a Miniaturized Six-Branch Hybrid Coupler
}

\author{
Changhong Zhang, Xianling Liang, Junping Geng, and Ronghong Jin \\ Department of Electronic Engineering, Shanghai Jiao Tong University, No. 800 Dongchuan Road, Shanghai 200240, China \\ Correspondence should be addressed to Xianling Liang; liangxl@sjtu.edu.cn
}

Received 22 July 2016; Accepted 8 September 2016

Academic Editor: Xiulong Bao

Copyright (c) 2016 Changhong Zhang et al. This is an open access article distributed under the Creative Commons Attribution License, which permits unrestricted use, distribution, and reproduction in any medium, provided the original work is properly cited.

\begin{abstract}
A broadband dual circularly polarized magnetoelectric dipole antenna (MEDA) fed by a miniaturized six-branch hybrid coupler (SBHC) is presented in this paper. First, a dual linearly polarized MEDA with a bandwidth of $73.3 \%$ is developed based on the previous design with a bandwidth of $52 \%$. The SBHC, with a miniaturized size of $53 \%$, is designed on a printed circuit board underneath the ground of the MEDA, which possesses an efficient bandwidth of $80.7 \%$ to generate the antenna for dual circular polarization. Measurement results show that the proposed dual circularly polarized MEDA achieves an impedance bandwidth of $84.5 \%$, an axial-ratio bandwidth of $81.8 \%$, and a nearly symmetrical, stable unidirectional radiation pattern with an average gain of $8 \mathrm{dBic}$ over its impedance bandwidth.
\end{abstract}

\section{Introduction}

Circularly polarized (CP) antennas have significance in modern wireless communications, such as satellite communication, global positioning system (GPS), radar system, radio frequency identification (RFID), wireless local area network (WLAN), and long term evolution (LTE), due to the flexibility without polarization mismatch, multipath fading, and faraday rotation effect. Dual CP antennas can realize both right hand circular polarization and left hand circular polarization, and thus they are valuable for the miniaturized systems with frequency reuse or polarization diversity. Broadband antennas represent the challenging issues for high-capacity communication, high-speed data-rate communication, or interoperating several systems within the same module. Therefore, broadband dual $\mathrm{CP}$ antennas are valuable and promising for the increasing requirements of wireless communications. In addition, the antennas with unidirectional, symmetrical, and stable radiation patterns are advantageous for such systems due to the high coverage efficiency.

Broadband dual CP antennas can be transformed from conventional broadband $\mathrm{CP}$ antennas. With reference to spiral antennas, many configurations have been designed for dual circular polarization at the expense of degraded array efficiency due to a halved effective use of space $[1,2]$, sacrificing gain by the resister load [3], and complex, bulky feeding networks $[4,5]$. Helix is another traditional broadband CP antenna and dual circular polarization can be generated through two ports in both ends, but orthogonal polarization operates at separate bands [6]. A dual CP slot antenna achieved a 3-dB axial-ratio (AR) bandwidth of approximately $60 \%$, within which return loss and isolation were better than $10 \mathrm{~dB}$ and $15 \mathrm{~dB}$, respectively, but with bidirectional radiation [7]. Patch antennas are generally narrow band radiators, few of which are developed to realize broadband dual CP antennas. A dual CP patch generated by cross slots via a transmission line underneath the ground plane attained a 3$\mathrm{dB}$ AR bandwidth of $48 \%$ and a $15-\mathrm{dB}$ return loss bandwidth of $60 \%$, at the cost of low isolation and gain [8]. Hybrid coupler is a popular scheme applied to patch antennas for wideband dual circular polarization. Nevertheless, the bandwidth of normal hybrid couplers is still limited. On the other hand, broadband multibranch hybrid couplers occupy a large area. Therefore, the hybrid couplers exceeding three branches are rarely adopted in antennas for dual circular polarization. A three-branch periodic hybrid coupler with an approximate $35 \%$ miniaturized size was designed to generate a circular patch for dual circular polarization, which 
obtained an overall bandwidth of $14.7 \%$ [9]. Consequently, it is necessary to develop a novel configuration rather than a traditional antenna for excellent performances of broadband dual circular polarization.

Magnetoelectric dipole antennas (MEDAs) with broadband and symmetrical, stable unidirectional radiation patterns have been widely investigated and some designs for circular polarization have been reported recently. A MEDA formed by two bowtie patch antennas and two electric dipoles achieved an impedance bandwidth of $41 \%$ and a $3-\mathrm{dB}$ AR bandwidth of $33 \%$ [10]. A crossed dipole loaded with a magnetoelectric dipole can exhibit an impedance bandwidth of $60 \%$ and a $3-\mathrm{dB}$ AR bandwidth of $27 \%$ [11]. An antenna composed of two cross-placed magnetoelectric dipoles and a feeding network comprising a wideband $90^{\circ}$ Schiffman phase shifter achieved a $10-\mathrm{dB}$ return loss bandwidth of $85.7 \%$ and a 3 -dB AR bandwidth of $61.5 \%$ [12]. A MEDA fed by a single $\Gamma$ shaped probe attained an impedance bandwidth of $73.3 \%$ and a 3-dB AR bandwidth of 47.7\% [13]. A dual linearly polarized (LP) MEDA, excited by a power divider and a broadband $90^{\circ}$ phase shifter, obtained an impedance bandwidth of $90 \%$ and a 3-dB AR bandwidth of $82 \%$ [14]. A $60 \mathrm{GHz}$ aperture coupled MEDA fed by a transverse slot etched on the broad wall of a section of shorted-end substrate integrated waveguide (SIW) showed an impedance bandwidth of $28.8 \%$ and a $3-\mathrm{dB}$ AR bandwidth of $25.9 \%$ [15]. However, these MEDA designs mentioned above are all based on single circular polarization.

In this paper, a broadband dual CP MEDA is proposed for the first time. The antenna is realized by a dual LP MEDA excited through a $3-\mathrm{dB}$ six-branch hybrid coupler (SBHC) with a $53 \%$ miniaturized size, which achieves an impedance bandwidth of $84.5 \%$ and a $3-\mathrm{dB}$ AR bandwidth of $81.8 \%$. Additionally, the antenna possesses a nearly symmetrical, stable unidirectional radiation pattern with an average gain of $8 \mathrm{dBic}$ across its impedance bandwidth. The remainder of this paper is organized as follows. Section 2 explains the basic design of the presented antenna. In Section 3, a prototype is fabricated and the performances are verified based on the experiment. Finally, the conclusion is given in Section 4.

\section{Broadband Dual CP MEDA Design}

The configuration of the dual CP MEDA is illustrated in Figure 1, which is composed of a dual LP MEDA and a miniaturized 3-dB SBHC. The SBHC is located below the ground of the dual LP MEDA, and the two output ports of the SBHC are connected to the $\Gamma$-shaped probes through the holes on the ground.

Figures 1(b), 1(c), and 1(d) show the configuration of the dual LP MEDA, which is constructed by four horizontally oriented square metallic plates, four vertically oriented shorted patches, two orthogonal $\Gamma$-shaped probes, and a square ground. The four square metallic plates, with the same side length $L_{d}$ and thickness $t_{d}$, are shorted to the ground through the vertical walls of the four shorted patches, with the length $L_{m}$, the width $W_{m}$, and the height $H_{m}$. Both the metallic plates and shorted patches are located uniformly, and they perform as crossed electric and magnetic dipoles
[14]. The two $\Gamma$-shaped probes are placed orthogonally in the gaps of the shorted patches. Each $\Gamma$-shaped probe, made by folding a straight metallic strip of rectangular cross-section into a $\Gamma$-shape, consists of three portions, namely, portion 1, portion 2, and portion 3, the corresponding function of which can be found in [16]. Compared to the dual LP MEDA introduced in [14], the antenna has three improvements on the $\Gamma$-shaped probes and ground, to broaden its bandwidth and make its structure symmetrical for broadband dual circular polarization. First, portion 2 of the $\Gamma$-shaped probes is placed parallel to the horizontally oriented metallic plates to introduce another resonance frequency for a broader impedance bandwidth, which will be detailed later. Second, the $\Gamma$-shaped probes are symmetrical with the exception of the lengths of portion 1, which have a slight difference equal to $t_{d}$. Nevertheless, the $\Gamma$-shaped probes in the previous design have different values in all dimensions, and five more variations are required. As a result, the antenna with symmetrical structure is more suitable to construct dual CP MEDA and has fewer variations for rapid optimization. Third, the four surrounding walls of the ground are removed, by which the antenna will have a lower quality factor and a wider bandwidth than the previous design.

Figures 1(e), 1(f), and 1(g) demonstrate the microstrip circuit of the miniaturized 3-dB SBHC, which consists of a thick ground, hollows, and microstrip lines from the bottom-up. The ground with thickness $t_{g}$ is located below the substrate. The rectangular hollows are placed underneath the stubs required high characteristic impedance in the SBHC design, with depths $t_{1}$ and $t_{2}$, lengths $L_{s_{1}}$ and $L_{s_{2}}$, and the same width $W_{s}$. The depths of the hollows are less than the thickness of the ground to reduce radiation losses. The main lines are all constructed by folded lines, with lengths of $L_{b n}$ $(n=1-3)$ and widths of $W_{b n}(n=1-3)$. By folding main lines, the total length of the whole SBHC can be shortened to $L_{c}$. The four ports of the SBHC are microstrip lines of $50 \Omega$, with a width of $W_{0}$. The substrate has a permittivity of 2.55 , loss tangent of 0.0018 , and thickness of $0.762 \mathrm{~mm}$.

2.1. Dual LP MEDA Design Concept and Parametric Study. MEDAs are more suitable for base stations, and the operating frequencies of the second-generation $(2 \mathrm{G}), 3 \mathrm{G}$, LTE mobile communications and handsets fall into the $0.698-2.65 \mathrm{GHz}$ frequency band [17]. The dual LP MEDA is designed to operate at the lower band. The antenna is investigated and optimized by CST microwave studio software, and the optimal parameters are listed in Table 1. Owning to the nearly symmetrical structure, only the antenna excited through the $\Gamma$-shaped probe with the lower height of $H_{f}$ is investigated for brevity.

To demonstrate the effect of paralleling the $\Gamma$-shaped probes to the horizontally oriented metallic plates, the reflection coefficient of the dual LP MEDA varying with the lower height $H_{f}$ of the $\Gamma$-shaped probes is illustrated in Figure 2, while the other parameters remain constant. It can be observed that the antenna has three resonance frequencies at approximately $0.75 \mathrm{GHz}, 1.1 \mathrm{GHz}$, and $1.4 \mathrm{GHz}$, when $H_{f}=H_{m}=68 \mathrm{~mm}$, with the $\Gamma$-shaped probes parallel to 


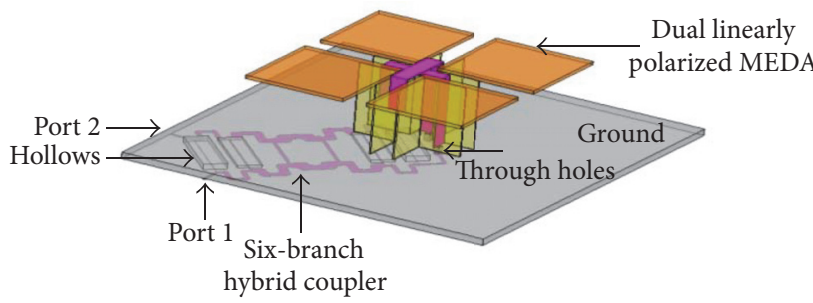

(a)

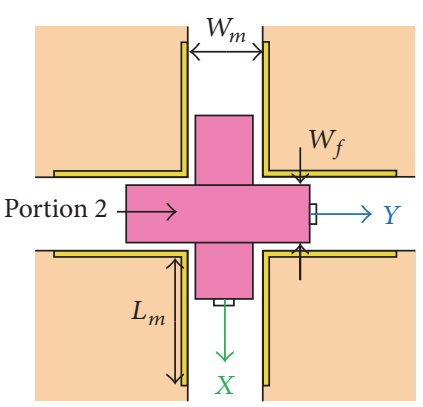

(b)

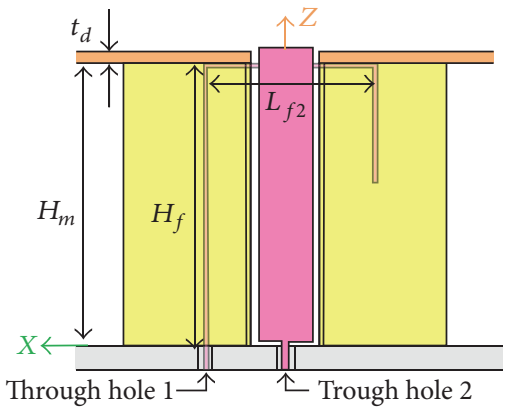

(c)

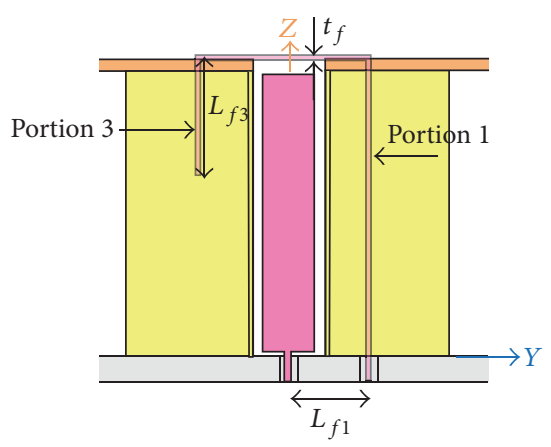

(d)

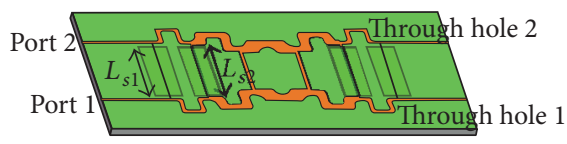

(e)

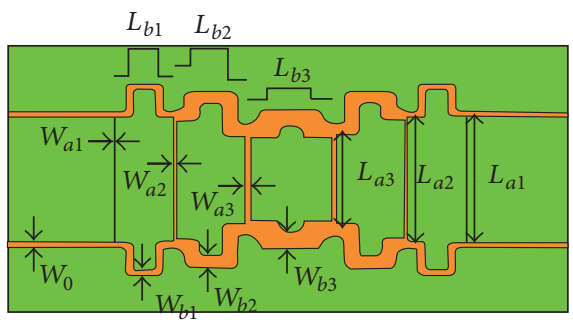

(f)

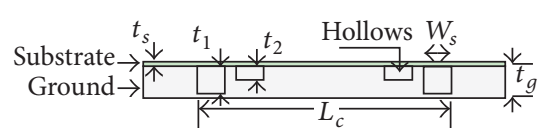

(g)

Figure 1: The configuration of the dual CP MEDA. (a) The 3D view of the dual CP MEDA. (b, c, and d) The top view, front view, and side view of the dual LP MEDA. (e, f, and g) The 3D view, top view, and sectional view of the 3 -dB SBHC.

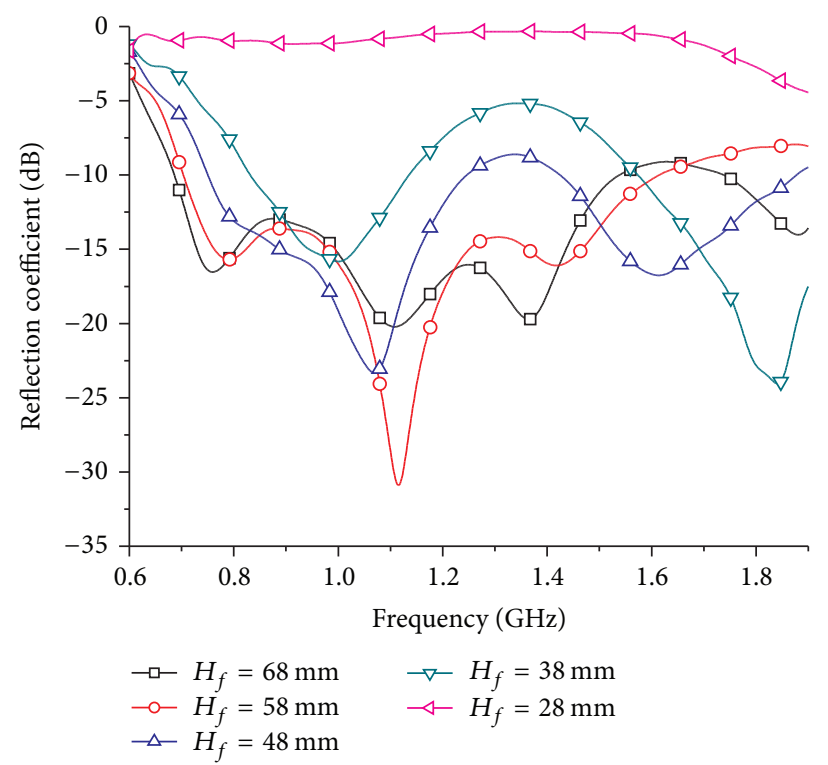

FIgURE 2: The reflection coefficient of the dual LP MEDA varies with the lower height $H_{f}$ of the $\Gamma$-shaped probes. 


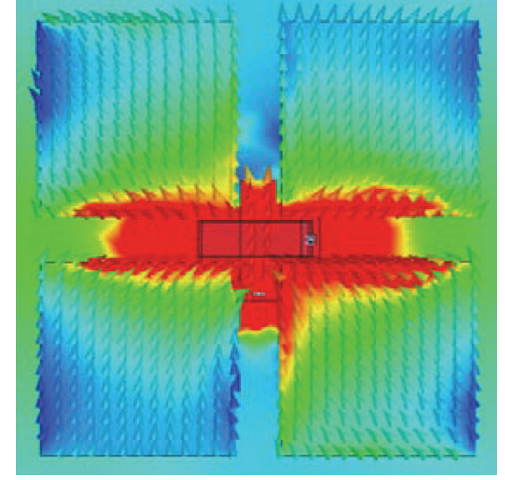

$t=0$

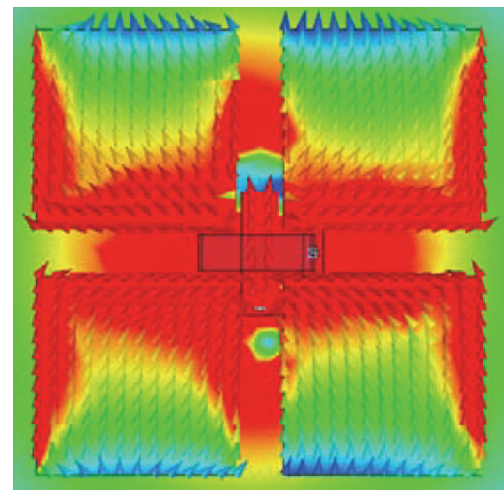

$t=T / 4$

(a)

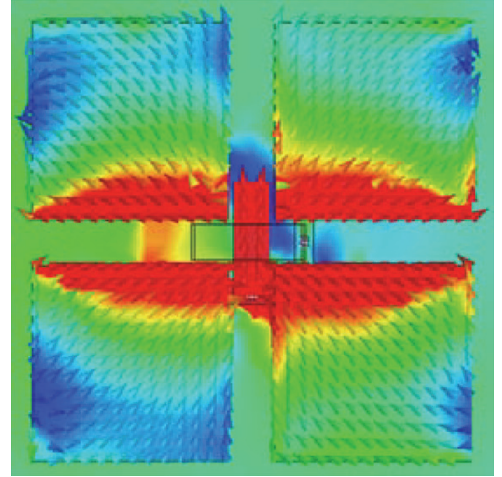

$t=0$

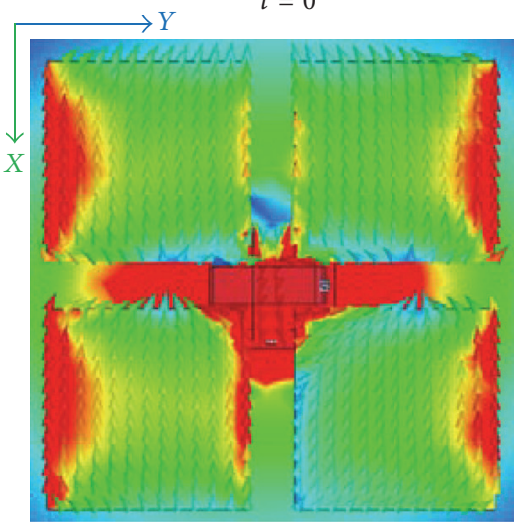

$t=T / 4$

(b)

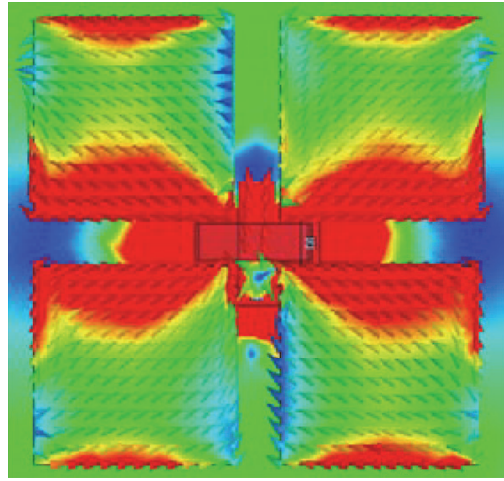

$t=0$

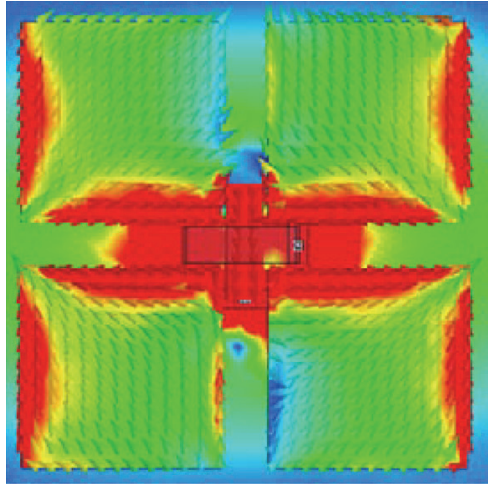

$t=T / 4$

(c)

Figure 3: The current distribution of the dual LP MEDA excited through the $\Gamma$-shaped probe with the lower height of $H_{f}$ at the three resonance frequencies. (a) $0.75 \mathrm{GHz}$. (b) $1.1 \mathrm{GHz}$. (c) $1.4 \mathrm{GHz}$.

TABLE 1: Optimized parameters of the dual CP MEDA.

\begin{tabular}{lccccccc}
\hline \multicolumn{7}{c}{ Parameters of the dual LP MEDA } \\
\hline Parameters & $L_{d}$ & $L_{m}$ & $L_{f_{1}}$ & $L_{f_{2}}$ & $L_{f_{3}}$ & $L_{g}$ & $W_{m}$ \\
Values/mm & 72 & 30 & 19.6 & 42 & 28.6 & 270 & 17 \\
Parameters & $W_{f}$ & $H_{m}$ & $t_{d}$ & $t_{f}$ & & & \\
Values/mm & 13 & 68 & 3 & 1 & & & \\
\hline \multicolumn{7}{c}{ Parameters of the 3-dB SBHC } \\
\hline Parameters & $W_{a_{1}}$ & $W_{a_{2}}$ & $W_{a_{3}}$ & $L_{a_{1}}$ & $L_{a_{2}}$ & $L_{a_{3}}$ & $L_{s_{1}}$ \\
Values/mm & 0.2 & 0.96 & 2.02 & 63.6 & 62.8 & 48.1 & 59.6 \\
Parameters & $W_{b_{1}}$ & $W_{b_{2}}$ & $W_{b_{3}}$ & $L_{b_{1}}$ & $L_{b_{2}}$ & $L_{b_{3}}$ & $L_{s_{2}}$ \\
Values/mm & 2.94 & 5.71 & 7.98 & 50.87 & 44.33 & 43.88 & 58.8 \\
Parameters & $W_{s}$ & $W_{0}$ & $t_{1}$ & $t_{2}$ & $t_{g}$ & $t_{s}$ & $L_{c}$ \\
Values/mm & 16 & 2.2 & 4.86 & 2.48 & 6 & 0.762 & 132.12 \\
\hline
\end{tabular}

the metallic plates. However, when $H_{f}$ is decreased to values lower than the height $H_{m}$ of the metallic plates in decrement of $10 \mathrm{~mm}$, the impedance bandwidth of the antenna is gradually narrowed. The lower resonance frequency at approximately $0.75 \mathrm{GHz}$ disappears and the higher resonance frequency at approximately $1.4 \mathrm{GHz}$ shifts past $1.6 \mathrm{GHz}$, when $H_{f}$ is less than $48 \mathrm{~mm}$. As a result, the antenna becomes the design given in [14], which has only two resonance frequencies and a narrower impedance bandwidth. The antenna achieves a simulated impedance bandwidth of $73.3 \%$ for the reflection coefficient less than $-10 \mathrm{~dB}$, covering the band from $0.68 \mathrm{GHz}$ to $1.54 \mathrm{GHz}$, whereas the previous design obtains a bandwidth of $52 \%$ for the SWR less than 2 .

To better understand the performances of the dual LP MEDA, the current distribution and important parameters including the side length $L_{d}$ of the metallic plates and the height $H_{m}$ of the shorted patches are analyzed by CST. The other parameters in Table 1 remain unchanged, while each individual parameter is varied.

Figure 3 displays the current distribution of the dual LP MEDA excited through the $\Gamma$-shaped probe with the lower height of $H_{f}$ at the three resonance frequencies. At the centre frequency of $1.1 \mathrm{GHz}$, the antenna operates as a perfect magnetoelectric dipole, with the magnetic and electric dipoles of nearly the same amplitude and a $90^{\circ}$ phase difference. However, the antenna works at a hybrid mode of electric and magnetic dipoles at the lower resonance frequency of $0.75 \mathrm{GHz}$, with the magnetic dipole excited at time $t=0$ and both the magnetic and electric dipoles generated at time $t=$ $T / 4$ ( $T$ is a period of time). This phenomenon also appears at the higher resonance frequency of $1.4 \mathrm{GHz}$, and higher modes are also generated at this frequency. Fortunately, the hybrid 


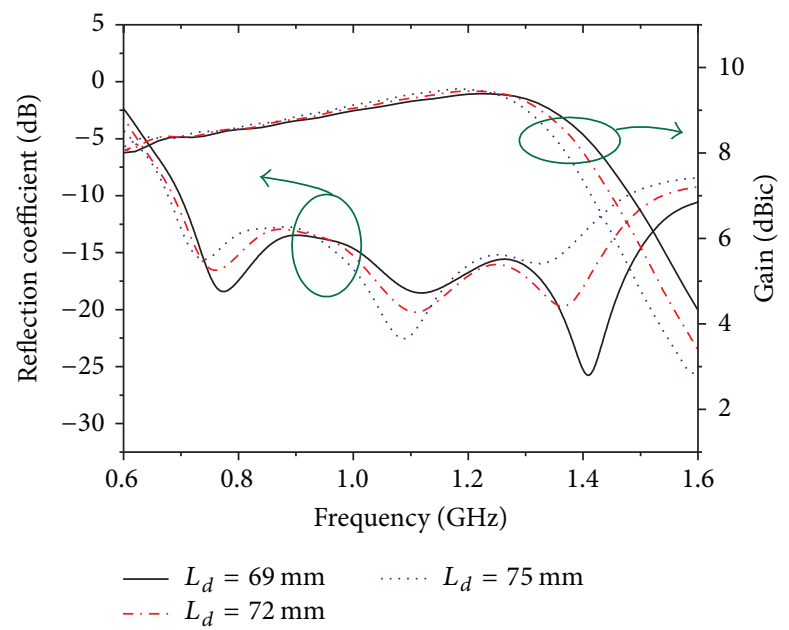

FIGURE 4: The reflection coefficient and gain of the dual LP MEDA vary with the side length $L_{d}$ of the metallic plates.

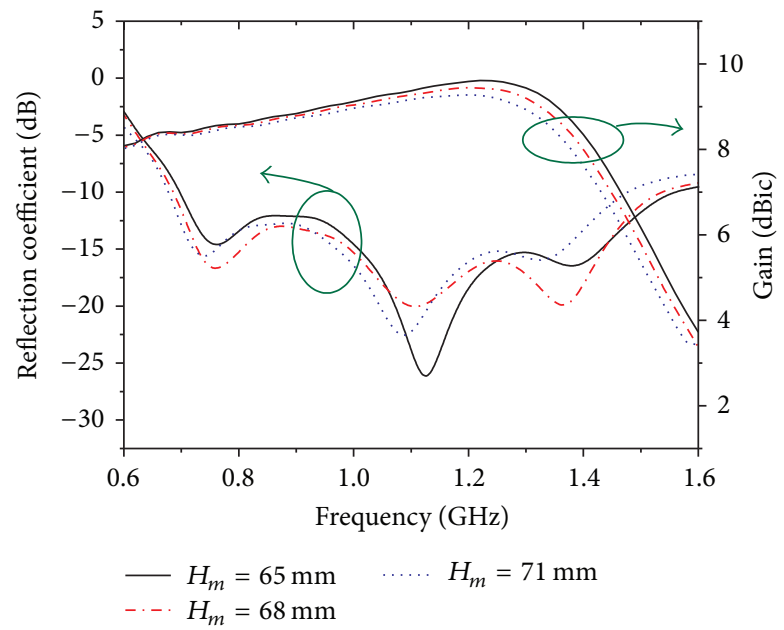

FIGURE 5: The reflection coefficient and gain of the dual LP MEDA vary with the height $H_{m}$ of the shorted patches.

mode of electric and magnetic dipoles still dominates at $1.4 \mathrm{GHz}$.

Figure 4 shows that the reflection coefficient and gain of the antenna vary with the side length $L_{d}$ of the metallic plates. Across the frequency band from $0.6 \mathrm{GHz}$ to $1.6 \mathrm{GHz}$, all the three resonance frequencies shift down as $L_{d}$ becomes longer. It can be observed that the reflection coefficient at the higher band is sensitive to $L_{d}$, because both the electric dipole and higher modes of the antenna are influenced by the length. Moreover, the gain reduces at the higher band while $L_{d}$ increases, which is primarily caused by the increasing higher modes. Considering the reflection coefficient, gain, and the associated efficient bandwidth, $L_{d}=72 \mathrm{~mm}$ is the optimal choice.

Figure 5 shows that the reflection coefficient and gain of the antenna vary with the height $H_{m}$ of the shorted patches, while keeping portion 2 of the $\Gamma$-shaped probes parallel to the metallic plates. The impedance bandwidth has limited change when $H_{m}$ varies from $65 \mathrm{~mm}$ to $68 \mathrm{~mm}$ but narrows significantly while $H_{m}$ approaches $71 \mathrm{~mm}$. The gain also reduces at the higher band while $H_{m}$ increases, but the range of change is smaller than that from varying the side length $L_{d}$ of the metallic plates. Considering the reflection coefficient, gain, and the associated bandwidth, $H_{m}=68 \mathrm{~mm}$ is selected.

The investigations above are summarized as follows. The impedance matching at the lower band can be broadened by paralleling the $\Gamma$-shaped probes to the metallic plates to introduce another resonance frequency. The impedance at the higher band is primarily determined by the side length $L_{d}$ of the metallic plates. The gain at the higher band rapidly drops, primarily due to the increasing higher modes generated on the metallic plates. It can be observed that the gain at the lower band slowly reduces, which is caused by the hybrid mode of the magnetic and electric dipoles with an imperfect combination [18].

2.2. Miniaturized 3-dB SBHC Design and Performances. Multibranch hybrid coupler is a conventional method to realize broadband hybrid coupler. The circuit model for 


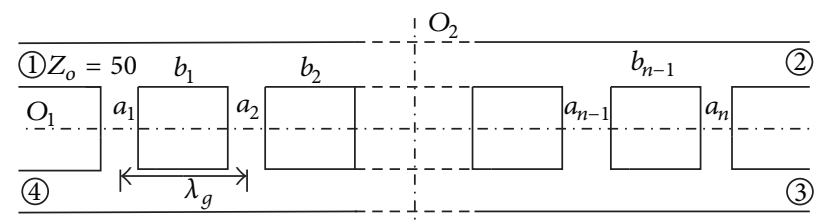

FIGURE 6: The circuit model of the multibranch hybrid coupler.

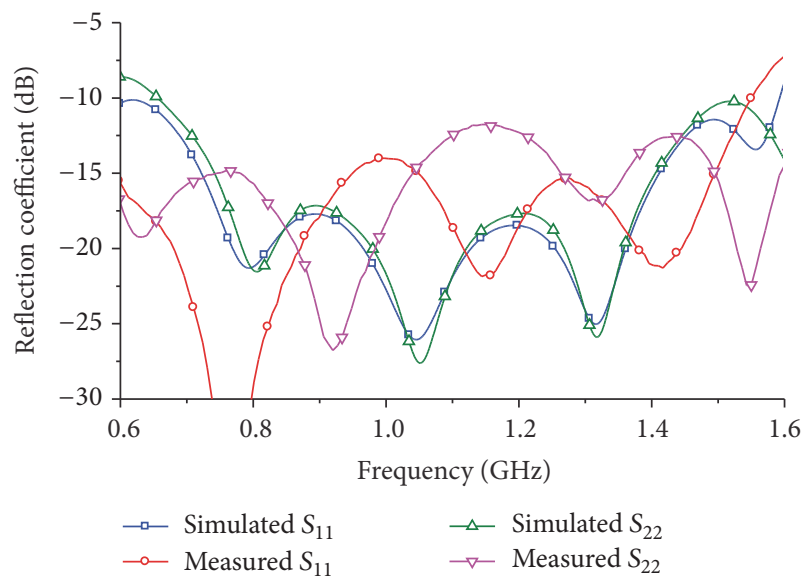

(a)

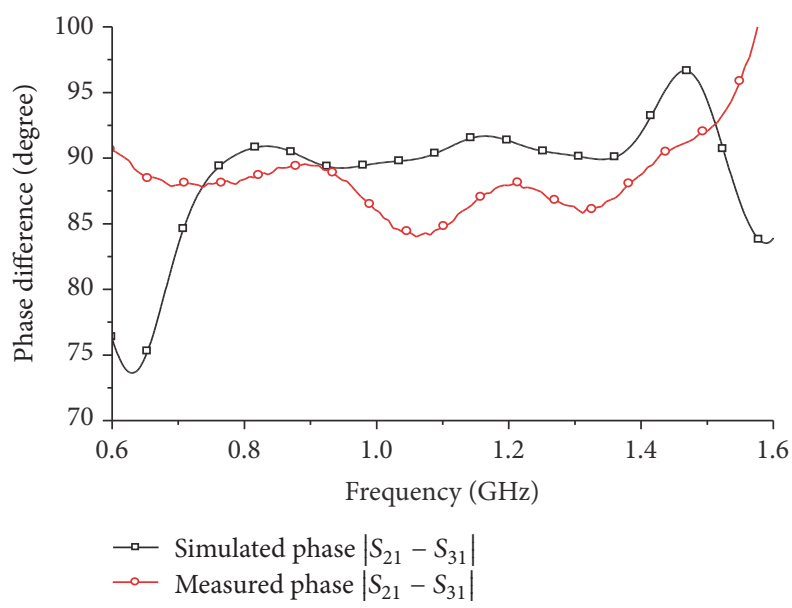

(c)

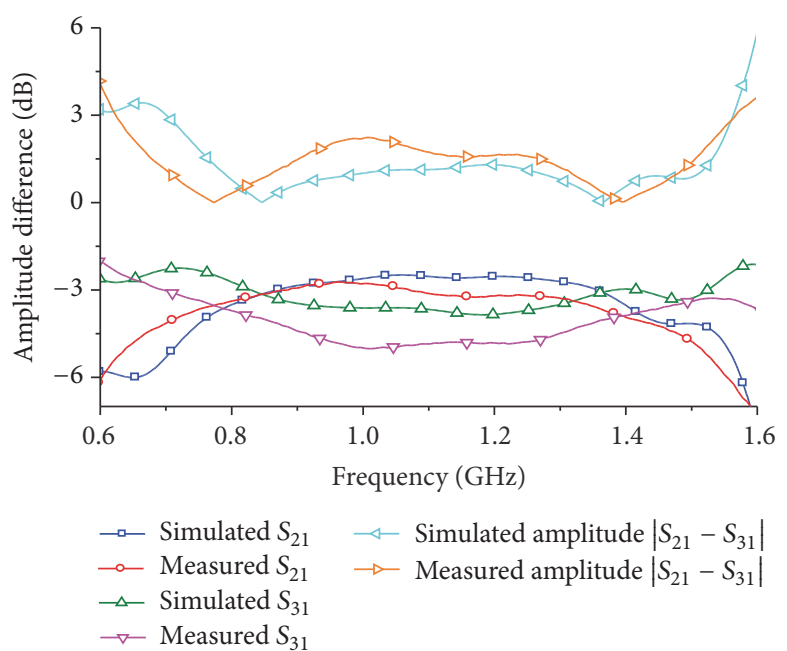

(b)

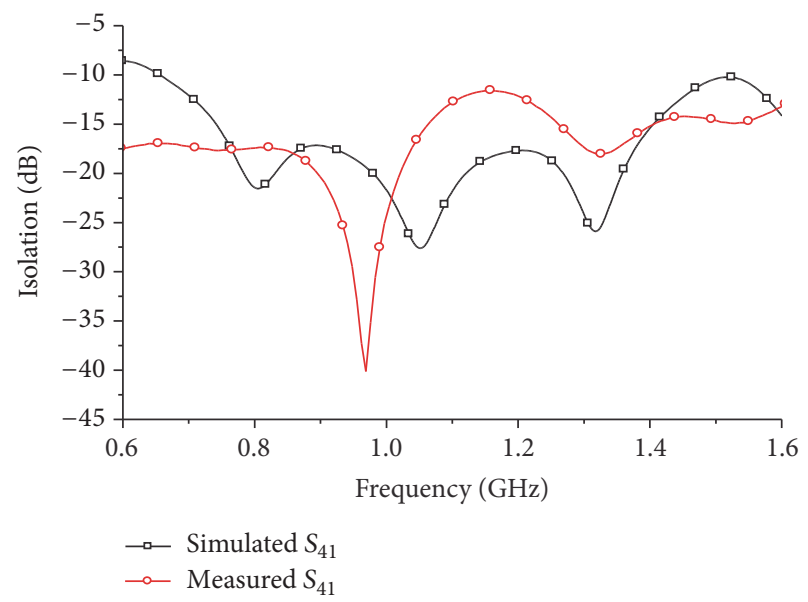

(d)

Figure 7: The $S$ parameter of the 3-dB SBHC. (a) The $S_{11}$ and $S_{22}$. (b) The amplitude of $S_{21}$ and $S_{31}$ and the amplitude difference between them. (c) The phase difference between $S_{21}$ and $S_{31}$. (d) The isolation between the two ports.

multibranch hybrid coupler is illustrated in Figure 6 [19]. The impedance of the stubs is donated by $a_{1}, a_{2}, \ldots, a_{n}$ and the main line impedance by $b_{1}, b_{2}, \ldots, b_{n}$. The lengths of each stub and main line are $\lambda_{g} / 4\left(\lambda_{g}\right.$ corresponds to the transmission line guide wavelength). Based on the synthesis method presented in [19], a symmetrical 3-dB SBHC is designed for the feeding circuit of the dual CP MEDA, and the theoretical characteristic impedance of the stubs and main lines is listed in Table 2.

In principle, the multibranch hybrid coupler can be designed on various types of transmission lines, whereas the one designed on printed circuit board (PCB) has become
TABLE 2: The theoretical characteristic impedance of the stubs and main lines of the 3-dB SBHC (unit: $\Omega$ ).

\begin{tabular}{ccccccc}
\hline & $a_{1}$ & $a_{2}$ & $a_{3}$ & $b_{1}$ & $b_{2}$ & $b_{3}$ \\
\hline$Z_{0}=50(\Omega)$ & 254 & 177 & 98 & 40 & 32 & 28 \\
\hline
\end{tabular}

main stream due to the advantages of low profile, simple fabrication, and low cost. In Table 2, it can be found that the maximum characteristic impedance of the stubs is $254 \Omega$, approximately 9 times the minimum of $28 \Omega$ of the main lines. Realizing high characteristic impedance is the most difficult 


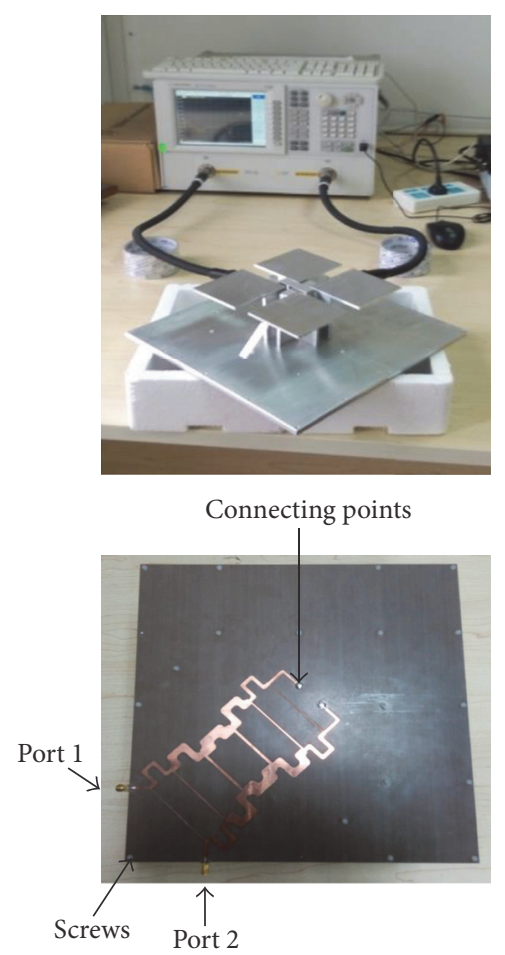

(a)
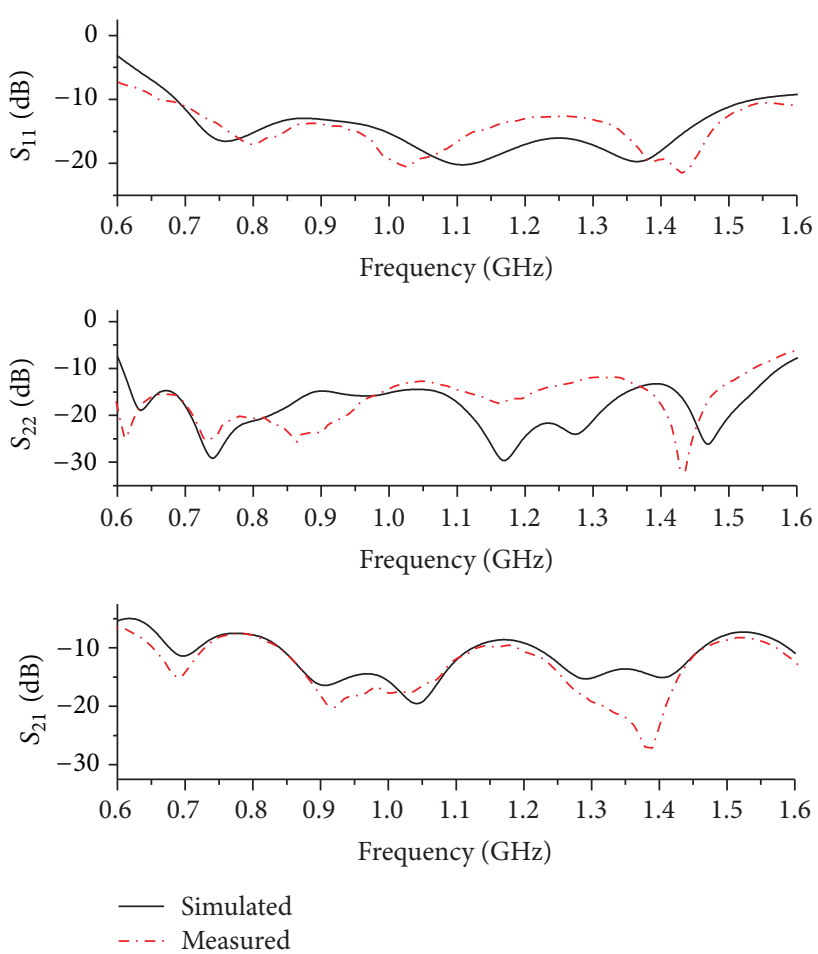

(b)

FIgURE 8: The pictures and $S$ parameter of the dual CP MEDA. (a) The pictures of the dual CP MEDA and 3-dB SBHC. (b) The $S$ parameter of the dual CP MEDA.

problem of the multibranch hybrid coupler designed on PCB. For high characteristic impedance, the ratio of the microstrip line width to the thickness of the substrate is required to be sufficiently small. The ultimate limit for normal PCB fabrication is $0.2 \mathrm{~mm}$ and the thickness of the substrate is also limited, which leads to the highest characteristic impedance realized by traditional PCB that is approximately $130 \Omega$ [20]. In this design, the rectangular hollows are placed underneath the stubs which required high characteristic impedance, to obtain the sufficiently small ratio of the microstrip line width to the thickness of the equivalent substrate.

Based on the theoretical characteristic impedance in Table 2, the SBHC is designed and optimized by CST, and the optimal parameters are listed in Table 1. The final length of the SBHC is $132 \mathrm{~mm}$, whereas the total length without folded lines is $250 \mathrm{~mm}$. As a result, the presented SBHC gains a miniaturized size of $53 \%$ through folding main lines. Moreover, with this method, the stubs with different widths $W_{a n}(n=1-3)$ can have different lengths of $L_{a n}(n=1-3)$ to achieve the identical electrical length of $\lambda_{g} / 4$. Therefore, improved performances of the SBHC can be achieved with folded lines.

The SBHC was fabricated and measured according to the parameters in Table 1 and the picture is shown in Figure $8(\mathrm{a})$. The simulated and measured $S$ parameter is shown in Figure 7. In Figure 7(a), the measured common reflection coefficient bandwidth of the two ports is $88.4 \%$ across the band from $0.6 \mathrm{GHz}$ to $1.55 \mathrm{GHz}$ for both $S_{11}$ and $S_{22}$ less than $-10 \mathrm{~dB}$ and covers the impedance bandwidth of the dual LP MEDA. Figure 7(b) shows the amplitude of
$S_{21}$ and $S_{31}$. The measured amplitude difference between $S_{21}$ and $S_{31}$ is less than $2.2 \mathrm{~dB}$ during the band from $0.65 \mathrm{GHz}$ to $1.53 \mathrm{GHz}$. Figure 7(c) shows the phase difference between $S_{21}$ and $S_{31}$, which is within $84^{\circ}-93.7^{\circ}$ from $0.6 \mathrm{GHz}$ to $1.53 \mathrm{GHz}$. In Figure 7(d), the measured isolation of $S_{41}$ is higher than $11.6 \mathrm{~dB}$ during the observation band from $0.6 \mathrm{GHz}$ to $1.6 \mathrm{GHz}$. Consequently, the SBHC obtains an efficient bandwidth of $80.7 \%$ from $0.65 \mathrm{GHz}$ to $1.53 \mathrm{GHz}$. The measured insertion loss of the SBHC is less than $0.68 \mathrm{~dB}$ over the efficient bandwidth. It should be noted that the emphases of the synthesis method and optimization adopted in this design are for the widest bandwidth and the minimum amplitude difference between $S_{21}$ and $S_{31}$ over the band, at the expense of the relatively lower isolation. The measured results have some divergences compared to the simulations, which are caused by fabrication errors, assembly errors, and inaccuracy in determining the permittivity of the substrate.

\section{Results}

The dual CP MEDA was fabricated and measured based on the parameters in Table 1 . The pictures of the dual CP MEDA and $3-\mathrm{dB}$ SBHC are shown in Figure 8(a). The simulation and measurement results including the $S$ parameter, radiation pattern, gain, and AR are compared and discussed.

Figure $8(\mathrm{~b})$ depicts the $S$ parameter of the dual CP MEDA. The measured reflection coefficient bandwidth of port 1 for $S_{11}$ less than $-10 \mathrm{~dB}$ is $88 \%$ within the band from $0.6 \mathrm{GHz}$ to $1.57 \mathrm{GHz}$, and the one of port 2 for $S_{22}$ 


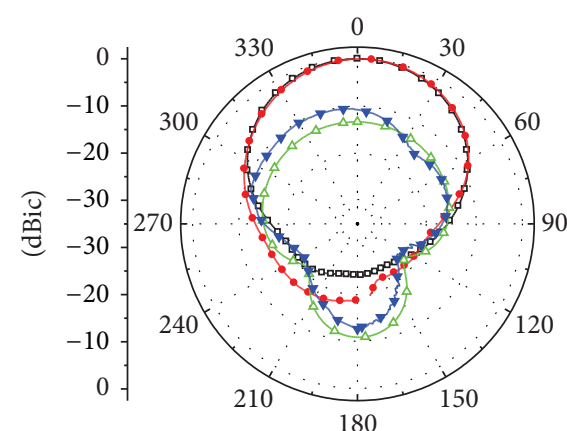

$0.7 \mathrm{GHz}\left(\mathrm{phi}=0^{\circ}\right)$

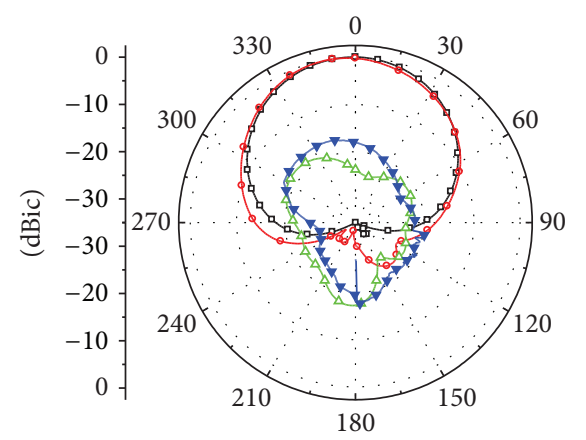

$1 \mathrm{GHz}\left(\mathrm{phi}=90^{\circ}\right)$

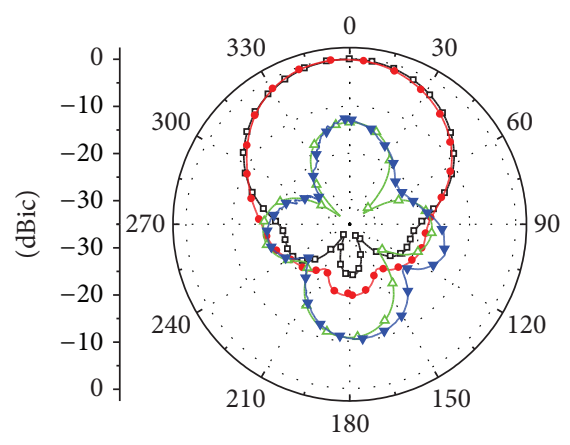

$0.7 \mathrm{GHz}\left(\mathrm{phi}=90^{\circ}\right)$

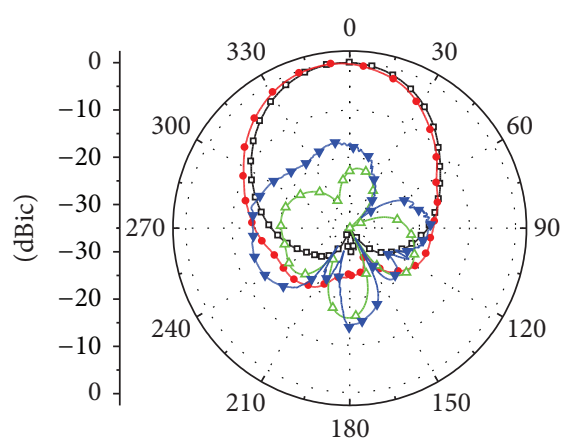

$1.3 \mathrm{GHz}\left(\mathrm{phi}=0^{\circ}\right)$

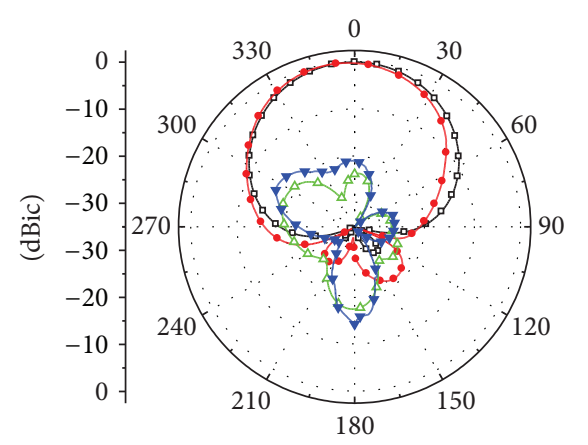

$1 \mathrm{GHz}\left(\mathrm{phi}=0^{\circ}\right)$

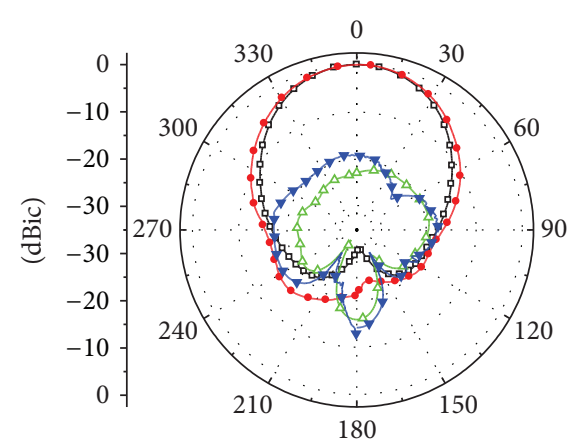

$1.3 \mathrm{GHz}\left(\mathrm{phi}=90^{\circ}\right)$

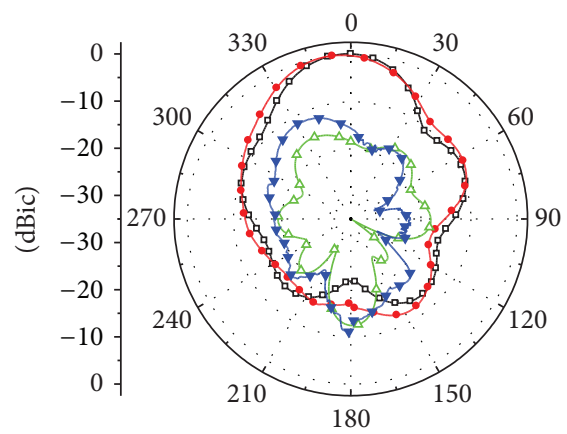

$1.5 \mathrm{GHz}\left(\mathrm{phi}=0^{\circ}\right)$

$\rightarrow$ Simulated copolarization
$\rightarrow$ Measured copolarization

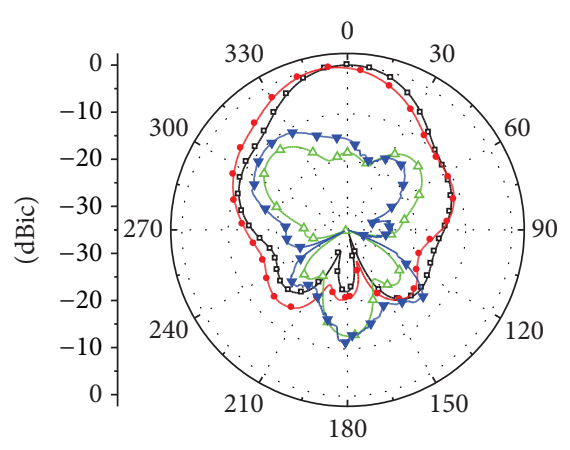

$1.5 \mathrm{GHz}\left(\mathrm{phi}=90^{\circ}\right)$

$\triangle-$ Simulated cross-polarization

$\rightarrow$ Measured cross-polarization

Figure 9: The normalized radiation patterns of the dual CP MEDA at the frequencies of $0.7 \mathrm{GHz}, 1 \mathrm{GHz}, 1.3 \mathrm{GHz}$, and $1.5 \mathrm{GHz}$.

less than $-10 \mathrm{~dB}$ is $84.5 \%$ across the band from $0.6 \mathrm{GHz}$ to $1.53 \mathrm{GHz}$. The measured isolation of $S_{21}$ between the two ports is greater than $6.6 \mathrm{~dB}$ during the observation band. The low isolation between the two ports is primarily due to two reasons. First, the isolation of the SBHC is only higher than $11.6 \mathrm{~dB}$ across the band. Second, the input impedance of the $\Gamma$-shaped probes is not exactly equal to $50 \Omega$ as the load of the SBHC, which will deteriorate the isolation. The simulated and measured results are well matched, except at a few frequencies. With reference to the requirements of a broadband antenna, those deviations are acceptable.

Figure 9 depicts the normalized radiation patterns of the dual CP MEDA in phi $=0^{\circ}$ and $\mathrm{phi}=90^{\circ}$ planes at the frequencies of $0.7 \mathrm{GHz}, 1 \mathrm{GHz}, 1.3 \mathrm{GHz}$, and $1.5 \mathrm{GHz}$.
The radiation patterns with a $3-\mathrm{dB}$ beamwidth of approximately $65^{\circ}$ remain nearly symmetrical and stable across the band except at $1.5 \mathrm{GHz}$, where slight deterioration occurs in both planes due to the higher modes. It can be observed that both the worst back radiation and cross-polarization are approximately $-10 \mathrm{~dB}$ at the lowest and highest frequency of $0.7 \mathrm{GHz}$ and $1.5 \mathrm{GHz}$, respectively. The higher back radiation at $0.7 \mathrm{GHz}$ is due to two reasons. First, the magnetic and electric dipoles with an imperfect combination form the hybrid mode [18]. Second, the ground has an electrical length that is only $0.63 \lambda_{o}$ ( $\lambda_{o}$ corresponds to the wavelength in air) at this frequency. At $1.5 \mathrm{GHz}$, the higher back radiation is caused by the spurious radiation from the SBHC below the ground. The lower cross-polarization at $0.7 \mathrm{GHz}$ and 


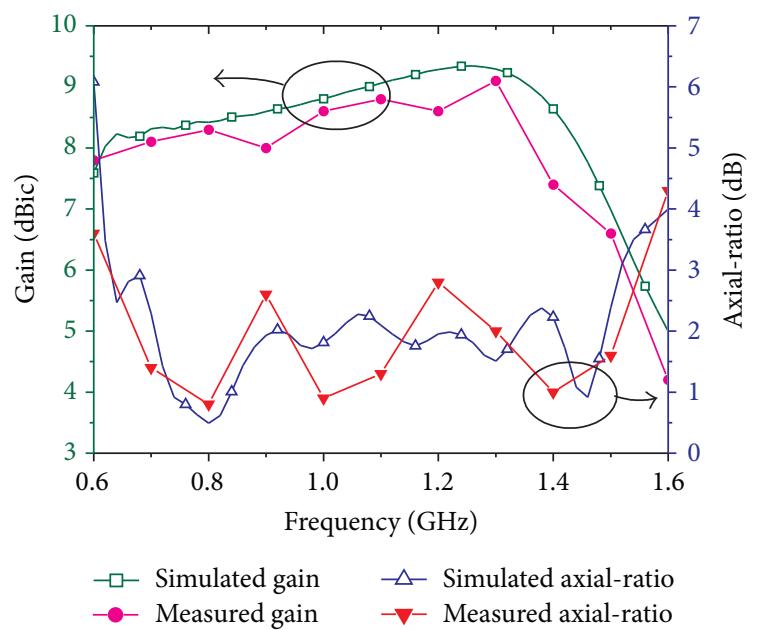

FIGURE 10: The gain and axial-ratio (AR) of the dual CP MEDA.

$1.5 \mathrm{GHz}$ is due to the imperfect feeding from the $3-\mathrm{dB}$ SBHC. The measured results are well matched with the simulations.

Figure 10 shows the gain and AR at the zenith of the dual CP MEDA. The gain is higher than $6 \mathrm{dBic}$ and has an average value of $8 \mathrm{dBic}$ within the impedance bandwidth from $0.6 \mathrm{GHz}$ to $1.53 \mathrm{GHz}$. The gain reaches a maximum of $9.1 \mathrm{dBic}$ at $1.3 \mathrm{GHz}$ and drops down when the frequency shifts past $1.3 \mathrm{GHz}$. That is due to the radiation pattern deteriorating while the frequency exceeds $1.3 \mathrm{GHz}$. The measured gain at some frequencies is lower than the simulation results, which is attributed to the losses not considered in the simulation. The measured 3-dB AR bandwidth at the zenith is $81.8 \%$ across the band from $0.63 \mathrm{GHz}$ to $1.53 \mathrm{GHz}$. The measured AR diverges simulation results over the band, which is due to the processing, assembly, and measurement errors.

\section{Conclusion}

In this work, a broadband dual CP MEDA is realized by a dual LP MEDA excited through a miniaturized 3-dB SBHC, which achieves an impedance bandwidth of $84.5 \%$ and a 3 - $\mathrm{dB}$ AR bandwidth of $81.8 \%$. By paralleling the $\Gamma$-shaped probes with the metallic plates, the bandwidth of the dual LP MEDA broadens largely through introducing another resonance frequency. The imperfect feeding from the $3-\mathrm{dB}$ SBHC deteriorates the cross-polarization of the antenna, which can be reduced by changing the square metallic plates to rectangular plates for fine-tuning. In addition, the methods to achieve high characteristic impedance and miniaturization adopted in the design of the SBHC can be used in other designs of multibranch hybrid couplers.

\section{Competing Interests}

The authors declare that there are no competing interests regarding the publication of this paper.

\section{Acknowledgments}

This work was supported by the National Natural Science Foundation $(61201058,61471240)$, the Research and Innovation Project of Shanghai Education Commission (12Z112030001), and the Scientific Research Foundation for Returned Overseas Chinese Scholars, State Education Ministry, and the Project of "SMC Excellent Young Faculty."

\section{References}

[1] I. D. Hinostroza Sáenz, R. Guinvarc'h, R. L. Haupt, and K. Louertani, "A dual-polarized wideband planar phased array with spiral antennas," IEEE Transactions on Antennas and Propagation, vol. 62, no. 9, pp. 4547-4553, 2014.

[2] M. Serhir and R. Guinvarc'H, "A low-profile cavity-backed dualpolarized spiral antenna array," IEEE Antennas and Wireless Propagation Letters, vol. 12, pp. 524-527, 2013.

[3] H.-T. Chou, P.-H. Hsueh, and L.-R. Kuo, "Two-arm spiral antenna array with dual circular polarised radiations for multibeam applications at Ku-band," Electronics Letters, vol. 48, no. 21, pp. 1322-1323, 2012.

[4] A. Mehrabani and L. Shafai, "Compact dual circularly polarized primary feeds for symmetric parabolic reflector antennas," IEEE Antennas and Wireless Propagation Letters, vol. 15, pp. 922-925, 2016.

[5] H. R. Fang, M. Serhir, R. Guinvarc'h, and K. Mouthaan, "Enhanced dual-circular polarised four-arm Archimedean spiral antenna with low-profile cavity backing," IET Microwaves, Antennas and Propagation, vol. 9, no. 12, pp. 1260-1266, 2015.

[6] Y. B. Jung, S. Y. Eom, S. I. Jeon, and S. O. Park, "Dualband and dual-polarised horn antenna including conical helix," Electronics Letters, vol. 43, no. 8, pp. 432-434, 2007.

[7] R. K. Saini and S. Dwari, "A broadband dual circularly polarized square slot antenna," IEEE Transactions on Antennas and Propagation, vol. 64, no. 1, pp. 290-294, 2016.

[8] E. Aloni and R. Kastner, "Analysis of a dual circularly polarized microstrip antenna fed by crossed slots," IEEE Transactions on Antennas and Propagation, vol. 42, no. 8, pp. 1053-1058, 1994.

[9] A. García-Aguilar, J.-M. Inclán-Alonso, L. Vigil-Herrero, J.-M. Fernández-González, and M. Sierra-Perez, "Low-profile dual circularly polarized antenna array for satellite communications in the X band," IEEE Transactions on Antennas and Propagation, vol. 60, no. 5, pp. 2276-2284, 2012.

[10] K. M. Mak and K. M. Luk, "A circularly polarized antenna with wide axial ratio beamwidth," IEEE Transactions on Antennas and Propagation, vol. 57, no. 10, pp. 3309-3312, 2009.

[11] S. X. Ta and I. Park, "Crossed dipole loaded with magnetoelectric dipole for wideband and wide-beam circularly polarized radiation," IEEE Antennas and Wireless Propagation Letters, vol. 14, pp. 358-361, 2015.

[12] W. Liang, Y.-C. Jiao, J. Li, and T. Ni, "Circularly polarised magneto-electric dipole antenna," Electronics Letters, vol. 50, no. 14, pp. 976-978, 2014.

[13] M. Li and K.-M. Luk, "A wideband circularly polarized antenna for microwave and millimeter-wave applications," IEEE Transactions on Antennas and Propagation, vol. 62, no. 4, pp. 18721879, 2014. 
[14] M. Li and K.-M. Luk, "Wideband magnetoelectric dipole antennas with dual polarization and circular polarization," IEEE Antennas and Propagation Magazine, vol. 57, no. 1, pp. 110-119, 2015.

[15] Y. Li and K.-M. Luk, "A 60-GHz wideband circularly polarized aperture-coupled magneto-electric dipole antenna array," IEEE Transactions on Antennas and Propagation, vol. 64, no. 4, pp. 1325-1333, 2016.

[16] K.-M. Luk and H. Wong, "A new wideband unidirectional antenna element," International Journal of Microwave and Optical Technology, vol. 1, no. 1, pp. 35-44, 2006.

[17] K.-M. Luk and B. Wu, "The magnetoelectric dipole-a wideband antenna for base stations in mobile communications," Proceedings of the IEEE, vol. 100, no. 7, pp. 2297-2307, 2012.

[18] D.-H. Kwon, "On the radiation Q and the gain of crossed electric and magnetic dipole moments," IEEE Transactions on Antennas and Propagation, vol. 53, no. 5, pp. 1681-1687, 2005.

[19] R. Levy and L. F. Lind, "Synthesis of symmetrical branch-guide directional couplers," IEEE Transactions on Microwave Theory and Techniques, vol. 16, no. 2, pp. 80-89, 1968.

[20] C.-W. Tang, M.-G. Chen, Y.-S. Lin, and J.-W. Wu, "Broadband microstrip branch-line coupler with defected ground structure," IEEE Electronic Letters, vol. 42, no. 25, pp. 1458-1460, 2006. 


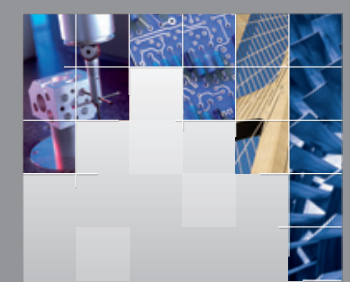

\section{Enfincering}
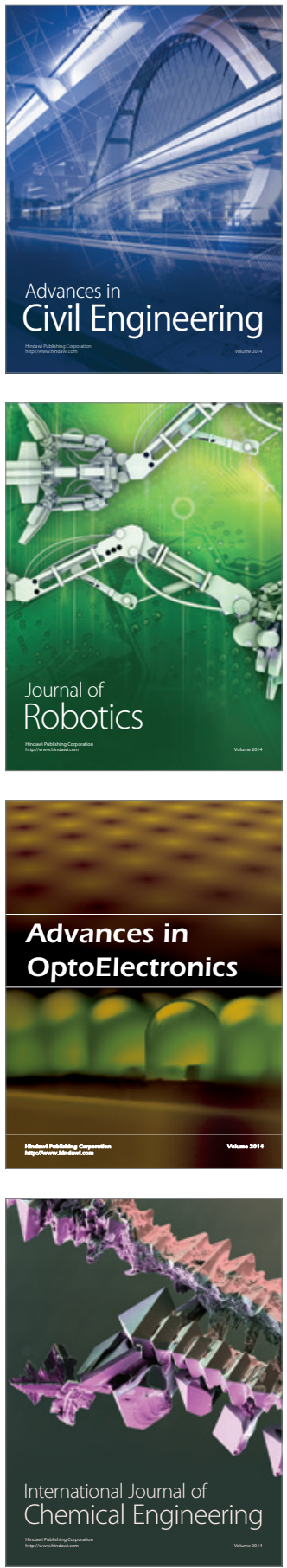

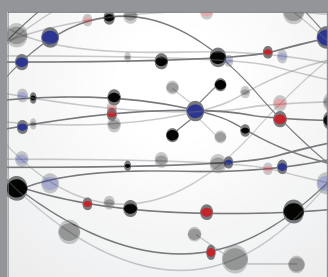

The Scientific World Journal

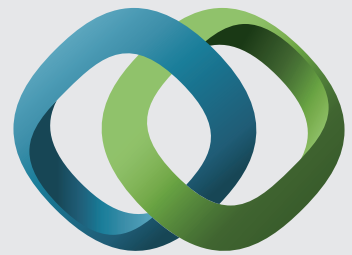

\section{Hindawi}

Submit your manuscripts at

http://www.hindawi.com
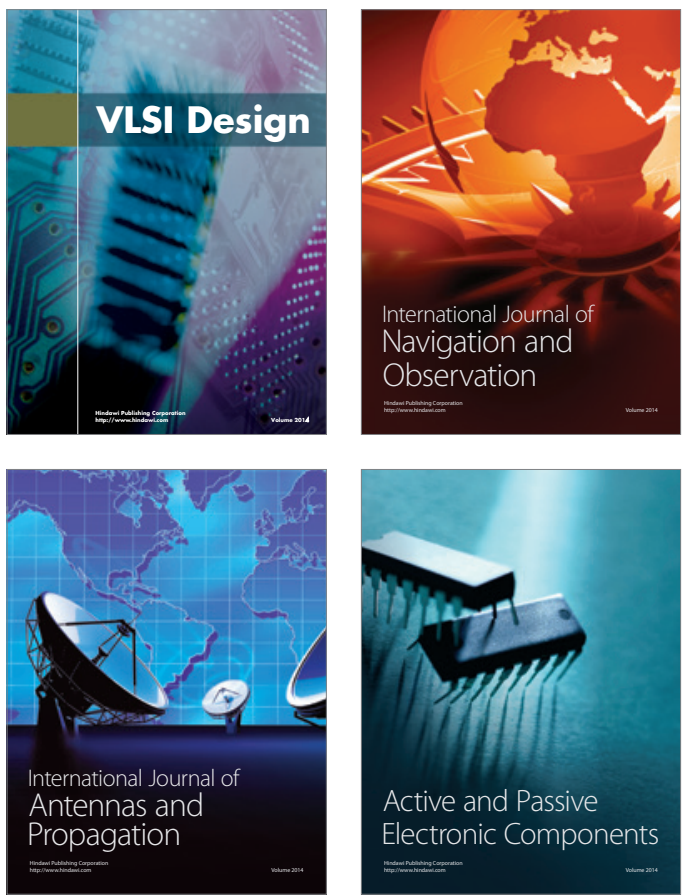
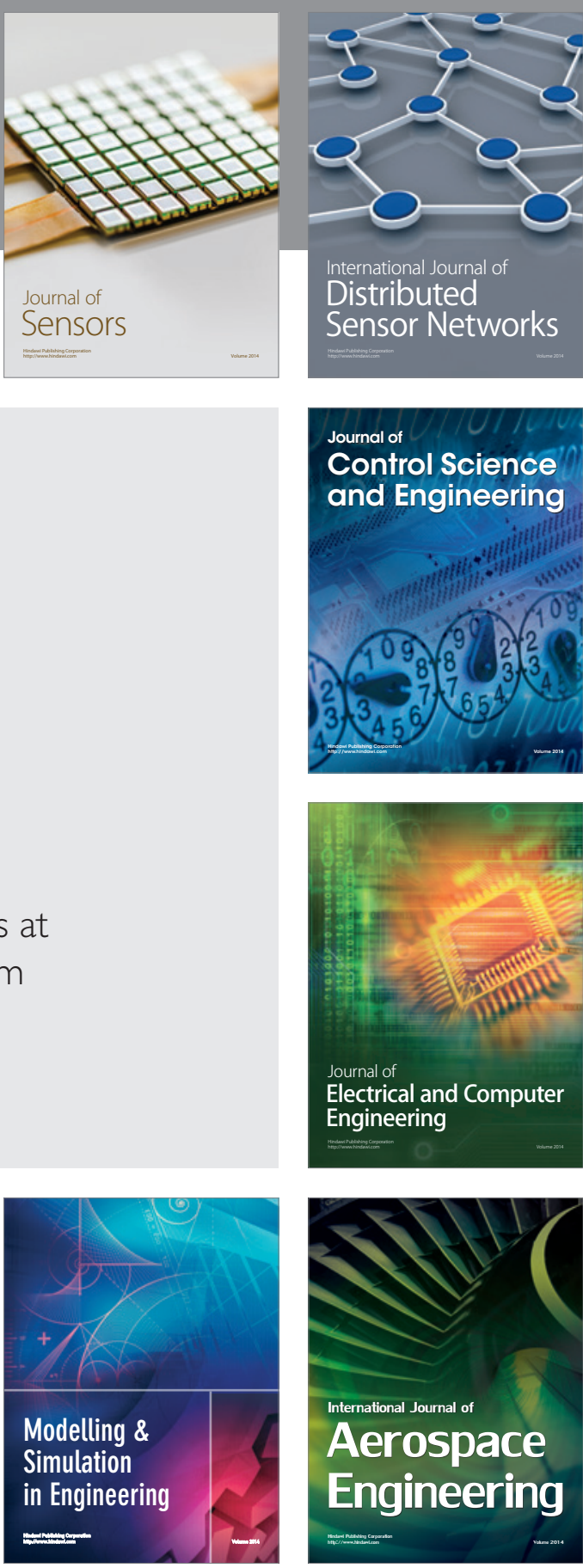

International Journal of

Distributed

Sensor Networks

Journal of

Control Science

and Engineering
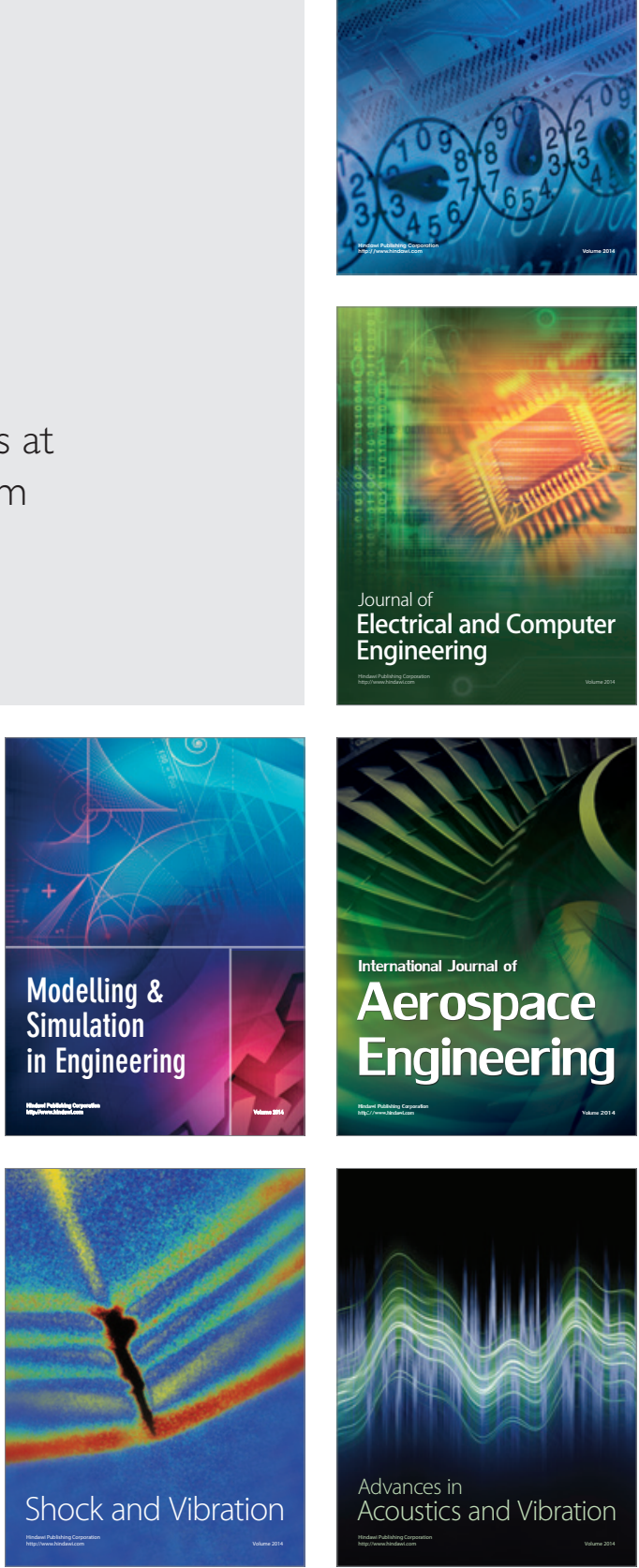